\title{
$\varepsilon$-Fructoselysine as an Absorption-delayed Material in the Small Intestinal Lumen of Rats Fed Browned Casein ${ }^{\dagger}$
}

\author{
Bunpei MorI \\ Laboratory of Food and Nutrition, Toita Women's College, Minato-ku, Tokyo 105
}

Received May 31, 1978

\begin{abstract}
Investigations were carried out in order to elucidate the reason for the nutritive value reduction of browned protein by using casein labeled with U-14 $\mathrm{C}$-L-lysine. When the browned casein was ingested by growing rats, high radioactivity was found in a lysine derivative present in the small intestinal TCA-soluble fraction obtained 3 and $7 \mathrm{hr}$ after feeding. Experiments were done to identify the lysine derivative as absorption-delayed material. This material was identified by an amino acid auto-analyzer, paper chromatography and radioactive analysis as 1-deoxy-1-( $\varepsilon-\mathrm{N}$-L-lysino)-D-fructose ( $\varepsilon$-fructoselysine), which accounted for about $70 \%$ of the total radioactivity in the small intestinal TCA-soluble fraction obtained $7 \mathrm{hr}$ after feeding. Lysine which was found after $3 \mathrm{hr}$, was disappeared from the intestinal TCA-soluble fraction taken $7 \mathrm{hr}$ after feeding, but $\varepsilon$-fructoselysine remainded. Its content as acetate found $7 \mathrm{hr}$ after one-dosal feeding of $600 \mathrm{mg}$ browned labeled casein $(700,000 \mathrm{dpm})$ was 18.3 and $19.0 \mathrm{mg} / \mathrm{each}$ rat, respectively. These values accounted for about $40 \%$ of the lysine content in the ingested browned casein.
\end{abstract}

In the previous report, ${ }^{1}$ ' distribution of radioactivities among the contents of digestive tract, urine and feces was determined after one dosal ingestion of the ${ }^{14} \mathrm{C}$-lysine-labeled casein, which was browned by amino-carbonyl reaction with glucose under a mild condition $\left(37^{\circ} \mathrm{C}\right.$ and R. H. $70 \%$ for 20 days). As the result, higher radioactivity was found in TCA-soluble fraction in the small intestinal lumen content as compared with that in the control group, while radioactivity was scarcely found in feces. Along with absorption delay, considerably high radioactivity was found in urine.

The limiting amino acid in browned casein prepared under mild condition was assumed to be lysine by estimating protein efficiency ratio. ${ }^{2)}$ Therefore, it was suggested that the reduction in nutritive value of casein by browning reaction may be mainly due to the formation of a lysine derivative in a protein, which remains in the small intestinal lumen as an

1 Studies on the Nutrition of Browned Protein. Part III. See ref. 1) for Part I and ref. 2) for Part II. This report was presented at the 10th International Congress of Nutrition, Kyoto, August, 1975.

Abbreviations: $\quad$ R. H., relative humidity; TCA, trichloroacetic acid. absorption-delayed material and is finally excreted in urine.

Present investigation was undertaken in order to identify the lysine derivative as an absorption-delayed material in the small intestinal lumen of rats under the same condition as in the previous report. ${ }^{1}$

\section{MATERIALS AND METHODS}

The preparation of browned casein labeled with U. ${ }^{14} \mathrm{C}$-L-lysine, the determination of the available lysine, and analysis of amino acid contents in browned and non-browned casein were the same as reported previously. ${ }^{1)}$ The radioactivity was measured with a liquid scintillation counter (Beckman LS-133).

1) Animals, Growing male Wistar rats (body weight $110 \sim 130 \mathrm{~g}$ ) were used. Diet components and the procedure of prefeeding were described previously. ${ }^{17}$

The experimental animals were given the diet for $3 \mathrm{hr}$ from $9.00 \mathrm{a} . \mathrm{m}$. each day by spaced feeding, and the prefeeding was continued for 3 weeks. In this experimental period the animals were trained to ingest the diet completely within $20 \mathrm{~min}$. After prefeeding, the animals were divided into 2 groups. One group was fed on labeled casein (non-browned) as the control, and the other on the browned labeled casein (as $600 \mathrm{mg}$ casein, $700,000 \mathrm{dpm}$ ) as the experimental group at 9.00 a.m. Labeled casein was given in a mixture with 


\section{$0.5 \mathrm{ml}$ of soybean oil.}

2) Isolation of an absorption-delayed material in the small intestinal content. Eight animals were divided into 2 groups, control and experimental groups of 4 rats each. Two animals from each group were killed by cutting out carotid after ether anesthesia at 3 and $7 \mathrm{hr}$ after labeled casein feeding. The content of the whole small intestine was taken out and homogenized with a Waring blender for $3 \mathrm{~min}$. The homogenate from each rat was treated separately in the method described below.

The homogenate was mixed with TCA-solution to give a final concentration (TCA) $7.5 \%$, followed by centrifugation at $12,000 \mathrm{rpm}$ for $20 \mathrm{~min}$. The supernatant was neutralized with $1 \mathrm{M} \mathrm{NaOH}$ to $\mathrm{pH} 5$, concentrated in vacuo at below $35^{\circ} \mathrm{C}$, and filled up with water to $50 \mathrm{ml}$. Twenty $\mathrm{ml}$ of this sample was subjected to gel filtration with a column $(4 \times 50 \mathrm{~cm})$ containing Sephadex G-25 (fine) by irrigating $0.05 \mathrm{M}$ acetic acid solution at a flow rate $50 \mathrm{ml} / \mathrm{hr}$, and eluates were fractionated in $8 \mathrm{ml}$ fractions.

Radioactivity was measured on each fraction obtained on Sephadex gel filtration, and fractions were analyzed by ninhydrin test according to the procedure of Yemm and Cocking. ${ }^{3)}$ Fractions which showed high radioactivity with positive ninhydrin reaction were collected and applied to a column $(1.7 \times 20 \mathrm{~cm})$ containing Dowex $50(w \times 8)$ resin in order to separate lysine derivatives. The column was irrigated with pyridine-acetic acid buffer, ${ }^{4} \mathrm{pH} 5.10$, at $58^{\circ} \mathrm{C}$.

Amino acids in the eluates were analyzed with a Shibata AA-100 type amino acid auto-analyzer fitted with a $15 \mathrm{~cm}$ column containing Aminex A-5 resin by irrigating with $0.35 \mathrm{M}$ citric acid buffer, pH 5.10 , at $58^{\circ} \mathrm{C}$.

3) Synthesis of 1-deoxy-I-( $\varepsilon-N-\mathrm{L}-l y$ sino $)$-D-fructose ( $\varepsilon$-fructoselysine).* The synthesis was carried out by the method of Finot and Mauron. ${ }^{\circ}$ )

4) Paper chromatography of e-fructoselysine. Paper chromatography was done by the procedure reported by Finot and Mauron. ${ }^{5}$ The solvent components are as follows: methyl ethyl ketone 50, acetic acid 10 and water 20 . Filter paper used was Toyo Filter Paper No. $51,(2 \times 40 \mathrm{~cm})$.

5) Measurement of radioactivity in each fraction. The measurement of the radioactivity was carried out by taking up $2 \mathrm{ml}$ of sample and mixed with $10 \mathrm{ml}$ of scintillator. ${ }^{* *}$ The measurement of the radioactivity after ninhydrin test, samples were oxidized with a

* It was delivered from K. Kusashio (Central research Lab., Ajinomoto Co.) to whom the author wishes to express sincere thanks.

** DPO $2 \mathrm{~g}$, POPOP $0.05 \mathrm{~g} /$ Toluene $500 \mathrm{ml}$, Triton $\times 100250 \mathrm{ml}$.
Packard oxidizer to ${ }^{14} \mathrm{CO}_{2}$ and the radioactivities were measured with a liquid scintillation counter in the above scintillator.

6) Separation and analysis of a lysine derivative with an amino acid auto-analyzer. The fractions which showed radioactivity on Sephadex gel filtration were collected and concentrated in vacuo at below $35^{\circ} \mathrm{C}$ and filled up with water in a volumetric flask.

The contents of lysine and a lysine derivative were determined on these samples with an amino acid autoanalyzer (Shibata AA-100 type) by Aminex A-5 resin with $0.35 \mathrm{M}$ citric acid buffer, $\mathrm{pH} 5.10$, at $58^{\circ} \mathrm{C}$.

\section{RESULTS AND DISCUSSION}

The amino acids composition and available lysine content of the browned and nonbrowned casein were reported in the previous paper. ${ }^{11}$

The optical densities of ninhydrin reaction at $570 \mathrm{~nm}$ and radioactivities in fractions eluted on Sephadex gel filtration of TCAsoluble fraction in the small intestinal content taken 3 and $7 \mathrm{hr}$ after browned casein feeding are shown in Figs. 1 and 2. Results on the

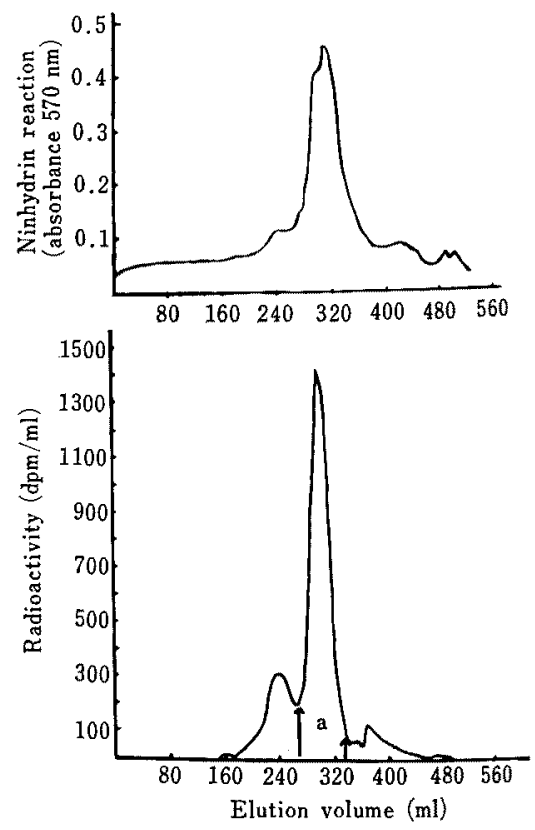

FIG. 1. Gel Filtration on Sephadex G-25 of TCAsoluble Fraction of the Intestinal Content Taken $3 \mathrm{hr}$ after the Browned Casein Feeding.

(Selected from typical experiments)

a. The part which was marked by arrows was taken to separate $\varepsilon$-fructoselysine. 

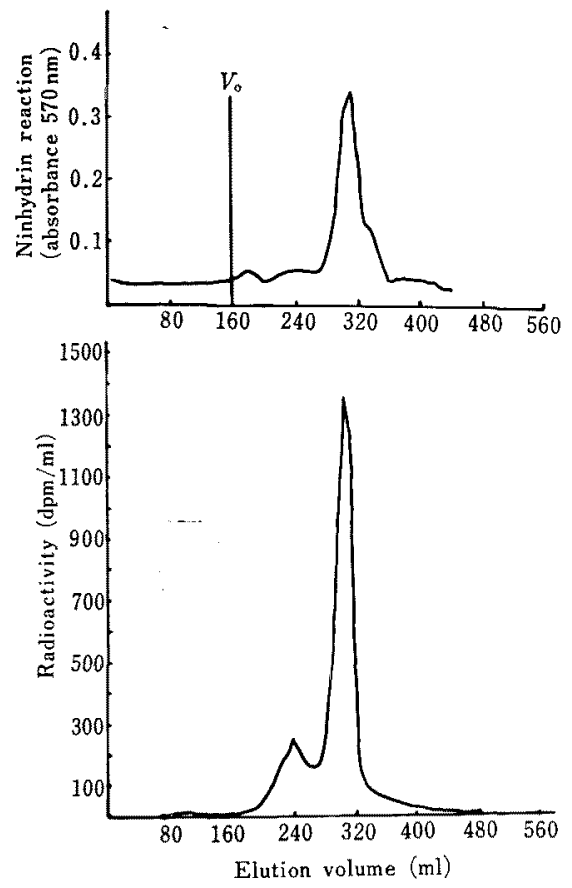

Fig. 2. Gel Filtration on Sephadex G-25 of TCAsoluble Fraction on the Intestinal Content Taken $7 \mathrm{hr}$ after the Browned Casein Feeding.

(Selected from typical experiments)

control (non-browned casein feeding) are shown in Figs. 3 and 4.

These results showed that, when the rats ingested the browned casein, higher radioactivity was found in the fractions eluted on
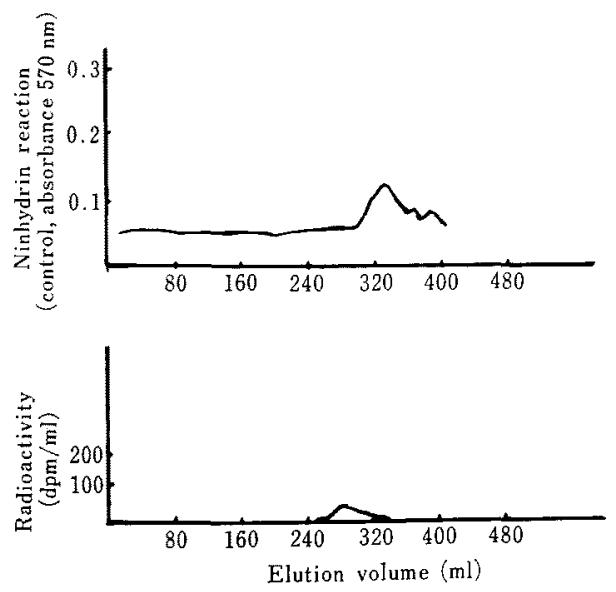

FIG. 3. Gel Filtration on Sephadex G-25 of TCAsoluble Fraction on the Intestinal Content Taken $3 \mathrm{hr}$ after the Non-browned Casein Feeding.

(Selected from typical experiments)
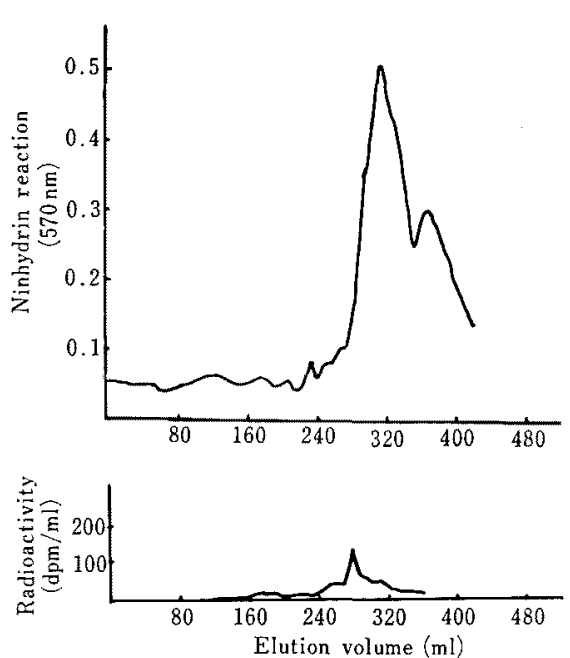

FIG. 4. Gel Filtration on Sephadex G-25 of TCAsoluble Fraction on the Intestinal Content Taken $7 \mathrm{hr}$ after the Non-browned Casein Feeding.

(Selected from typical experiments)

Sephadex gel filtration of TCA-soluble fraction in the small intestinal content, as compared with the control group. The radioactive peak and the peak in ninhydrin reaction appeared at the same position on Sephadex gel filtration (Figs. 1 and 2). As to the control group, radioactive peak was scarcely found in the small intestinal TCA-soluble fraction (Figs. 3 and 4). This difference between the experimental and the control groups coincided with the result observed under the same condition in the previous paper. ${ }^{11}$

The material, which holds high radioactivity with positive ninhydrin reaction in the small intestinal TCA-soluble fraction after browned labeled casein feeding, was suggested to be a lysine derivative from results mentioned below. After hydrolysis of this fraction with $6 \mathrm{~N} \mathrm{HCl}$ at $110^{\circ} \mathrm{C}$ for $24 \mathrm{hr}$, the presence of free lysine was confirmed with an amino acid autoanalyzer, and high radioactivity in this lysine derivative was shown by radioactive analysis as ${ }^{14} \mathrm{CO}_{2}$ after oxidation with a Packard oxidizer (Fig. 5). Then, highly radioactive fractions which were separated from the eluate (marked by arrows on Fig. 1), were concentrated in vacuo at below $35^{\circ} \mathrm{C}$. This concentrated fraction with high radioactivity was 


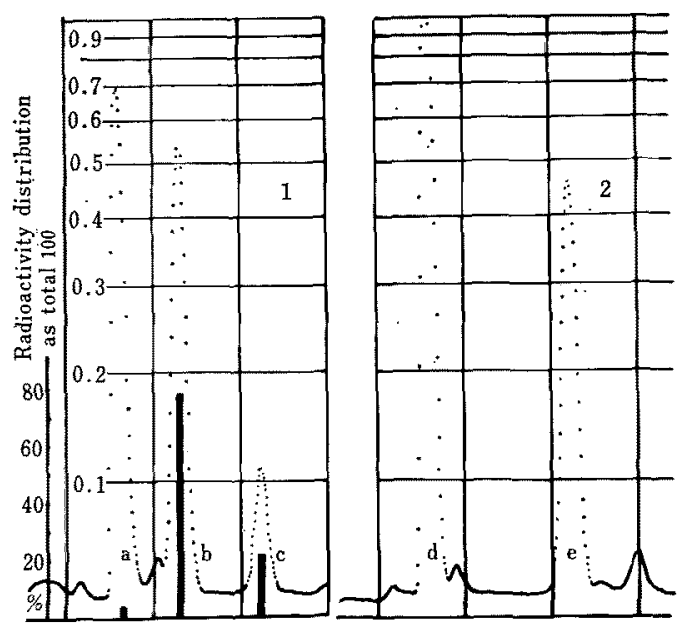

Fig. 5. The Chromatogram of a Lysine Derivative.

(Selected from typical experiments)

$15 \mathrm{~cm}$ column containing Aminex A-5 resin, citric acid buffer $\mathrm{pH} 5.10,58^{\circ} \mathrm{C}$.

1. Chromatogram of the high radioactive fraction obtained Sephadex gel filtration of TCA-soluble fraction of the intestinal content ( $3 \mathrm{hr}$ after feeding), and its radioactivity distribution (sample 1).

a: Other amino acids

b: Lysine derivative

c: Lysine

2. Hydrolysis of sample 1 with $6 \mathrm{~N} \mathrm{HCl}$ at $110^{\circ} \mathrm{C}$ for $24 \mathrm{hr}$.

d: Other amino acids

e: Lysine

further purified with a $1.7 \times 20 \mathrm{~cm}$ column containing Dowex $50(w \times 8)$ resin by irrigating with pyridine-acetic acid buffer, ${ }^{4}$ pH 5.10 at $58^{\circ} \mathrm{C}$. The separation of lysine and a lysine derivative with Dowex 50 were not complete, but this fraction was separated into single peaks of lysine and lysine derivative with an amino acid auto-analyzer using Aminex A-5 resin (Fig. 6).

Then, recovery test was carried out to identify, on an amino acid auto-analyzer, a lysine derivative which was purified on a Dowex 50 resin column. Synthetic $\varepsilon$-fructoselysine was used as the standard. The chromatogram is shown in Fig. 6, and the recovery of the standard in the recovery test was $96.8 \%$, as shown in Table I. The $R f$ value of a lysine derivative in paper chromatography coincided with that of synthesized $\varepsilon$-fructoselysine as

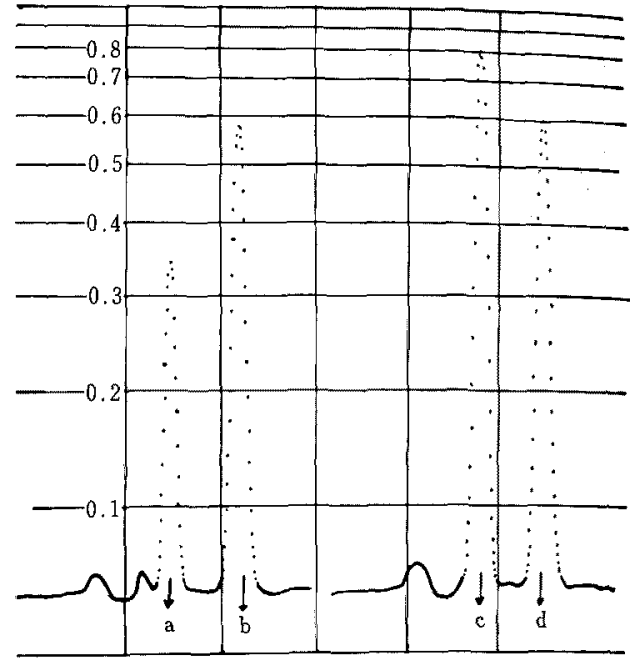

FIG. 6. The Chromatogram of the Separated $\varepsilon$ Fructoselysine and the Recovery Test on an Amino Acid Auto-analyzer.

$15 \mathrm{~cm}$ column containing Aminex A-5 resin, citric acid buffer, $\mathrm{pH} 5.10,58^{\circ} \mathrm{C}$.
a: e-Fructoselysine
$91.5 \mu \mathrm{g}$
b: Lysine
$36.6 \mu \mathrm{g}$
c: Separated sample $+\varepsilon$-fructoselysine
$91.5 \mu \mathrm{g}$
d: Lysine
$36.6 \mu \mathrm{g}$

Table I. The Recovery $\%$ of Lysine Derivative in Fig. 6

\begin{tabular}{ccc}
\hline $\begin{array}{c}\text { The amount of } \\
\text { synthetic } \varepsilon- \\
\text { fructoselysine } \\
\text { added }(\mu \mathrm{g})\end{array}$ & $\begin{array}{c}\varepsilon \text {-Fructoselysine } \\
\text { content } \\
\text { determined } \\
(\mu \mathrm{g} / 0.05 \mathrm{ml} \text { sample })\end{array}$ & Recovery \\
$(\%)$ \\
\hline- & 150.4 & \\
91.5 & 234.2 & 96.8 \\
\hline
\end{tabular}

Table II. $R f$ Values Paper Chromatography of the Separated Lysine Derivative and SYNTHETIC $\varepsilon$-Fructoselysine

$\begin{array}{lc}\text { Separated sample } & R f \\ \varepsilon \text {-Fructoselysine } & 0.23 \\ \text { Lysine } & 0.23 \\ \end{array}$

\section{shown in Table II.}

From these results, the lysine derivative which was separated from the small intestinal TCA-soluble fraction was identified as $\varepsilon$ fructoselysine. Amino acid residues in casein are partially changed to the nutritionally inactive forms by the amino carbonyl reaction, and the amino acid which is mostly influenced 
by this process may be lysine. ${ }^{6)}$ From our results using the ${ }^{14} \mathrm{C}$-labeled casein, $\varepsilon$-fructoselysine was an absorption-delayed material which was formed from available lysine in casein. In the fraction of ninhydrin peak on Sephadex gel filtration of the control, no $\varepsilon$ fructoselysine with radioactivity was found.

According to our results, ${ }^{1,7)} \varepsilon$-fructoselysine was not utilized as body protein accompanying with absorption delay, and it was excreted in urine for $22 \mathrm{hr}$ after feeding.

The fraction showing the peak in ninhydrin reaction on Sephadex gel filtration of TCAsoluble fraction of intestinal content taken $3 \mathrm{hr}$ after browned casein feeding was almost identical with that of the radioactive peak. Then, the fraction, which was marked by arrows on Fig. 1, was separated directly from the elutate on Sephadex gel filtration, and concentrated in vacuo at below $35^{\circ} \mathrm{C}$. The concentrated samples were filled up to $10 \mathrm{ml}$ and analyzed with an amino acid auto-analyzer. A typical chromatogram is shown in Fig. 7.

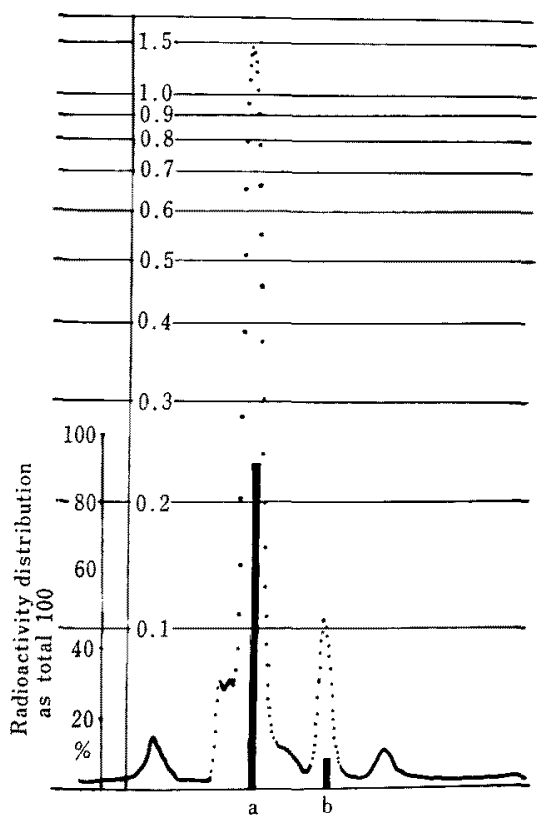

FIG. 7. The Chromatogram and Radioactivity Distribution of the Concentrated Radioactive Fraction Taken 3 hr after the Browned Casein Feeding.

a: $\varepsilon$-Fructoselysine

b: Lysine
The major component of the fraction was $\varepsilon-$ fructoselysine, whereas a small amount of lysine was also found in samples taken $3 \mathrm{hr}$ after browned casein feeding (Fig. 7). But, after $7 \mathrm{hr}$, lysine was not found. The endogenous lysine might be included in the amount of lysine, which was found in the intestinal TCA-soluble fraction after $3 \mathrm{hr}$. From chromatogram on Fig. 7, two peaks ( $\varepsilon$-fructoselysine and lysine) were separated, and the distribution of radioactivity of the peaks was measured with a Packard oxydizer as ${ }^{14} \mathrm{CO}_{2}$.

As a results, the ratio of distribution of radioactivity in $\varepsilon$-fructoselysine and lysine of the small intestinal TCA-soluble fraction $3 \mathrm{hr}$ after browned casein feeding under our experimental conditions, was 92 to 8 (Fig. 7).

Figure 8 shows the content of $\varepsilon$-fructoselysine was carried out with an amino acid autoanalyzer for each fraction on Sephadex gel filtration, by using the standard solution of synthetic $\varepsilon$-fructoselysine.

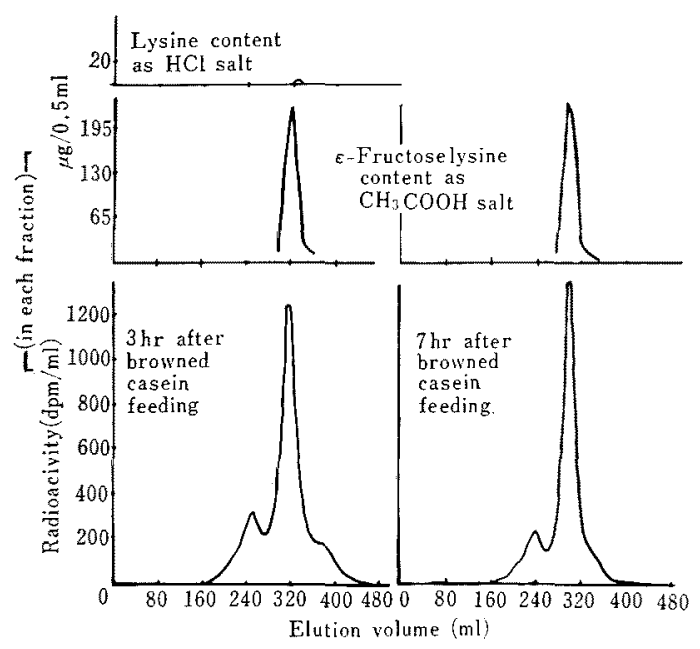

Fig. 8. The Contents of $\varepsilon$-Fructoselysine in Each Fraction with Radioactivity Taken 3 and $7 \mathrm{hr}$ after the Browned Casein Feeding.

(Selected from typical experiments)

The result, indicated that, the major part of the highly radioactive material consists of $\varepsilon$ fructoselysine. Whereas lysine was found after $3 \mathrm{hr}$, it was disappeared from the intestinal TCA-soluble fraction $7 \mathrm{hr}$ after feeding. This 
fact suggested that lysine was absorbed from intestinal mucosa, but $\varepsilon$-fructoselysine remained in the intestinal lumen. $\varepsilon$-Fructoselysine content in the small intestinal TCAsoluble fraction was determined with an amino acid auto-analyzer. Its content as acetate found $7 \mathrm{hr}$ after one dosal feeding of $600 \mathrm{mg}$ browned labeled casein was 18.3 and $19.0 \mathrm{mg}$ in each rat, respectively. These values accounted for about $40 \%$ of the lysine content in the ingested browned casein.

According to Erbersdobler, ${ }^{8)} \varepsilon$-fructoselysine is not actively transported out of the intestine. An uptake by diffusion, however, is possible. Compared to lysine, $\varepsilon$-fructoselysine is absorbed at a slower rate. The result in the present paper is in good agreement with the data of Erbersdobler.

About $70 \%$ of total radioactivity in the eluted fractions on Sephadex gel filtration $7 \mathrm{hr}$ after feeding was contained in $\varepsilon$-fructoselysine. But, at $3 \mathrm{hr}$ after feeding, about $6 \%$ of the radioactivity was in lysine, and about $16 \%$ of the total radioactivity in the eluted fraction was in the parts which show no ninhydrin reaction. The ninhydrin-negative fraction always appeared preceding to $\varepsilon$-fructoselysine on Sephadex gel filtration (Fig. 1). There may be some substances which carbon chain derived from lysine as Maillard reaction product or a deamination product formed by intestinal microflora. Erbersdobler, Gunsser and Weber ${ }^{9}$ reported that the deamination of $\varepsilon$-fructoselysine occurs by the small intestinal microflora.

When non-browned casein was ingested as the control, radioactivities in each separated fraction were low, and lysine was found, but no $\varepsilon$-fructoselysine was found in any fraction on Sephadex gel filtration. Finot ${ }^{10)}$ proposed to use $\varepsilon$-fructoselysine as an index in determining the availability of lysine.

In our data published elsewhere, ${ }^{2)}$ protein efficiency ratio (PER) of browned casein was decreased, and it was recovered to the original level by the addition of equal amount of lysine to that, which was lost by browning reaction. Therefore, the limiting amino acid in browned casein was assumed to be lysine. The coefficient of true digestibility was not decreased.

From results described paper, a lysine derivative which remained as a absorption-delayed material in the small intestinal lumen of rats was proved to be $\varepsilon$-fructoselysine. It was accounted for about $70 \%$ of the total radioactivity in the small intestinal TCA-soluble fraction remained at $7 \mathrm{hr}$ after browned casein feeding.

Acknowledgement. The author wishes to express sincere thanks to Dr. H. Naito, Department of Agricultural Chemistry, The University of Tokyo, Dr. T. Kobayashi, Department of Agricultural Chemistry, Tokyo Noko University, and Central Research Laboratories, Ajinomoto Co., Ltd., for the interest in and support of this study.

\section{REFERENCES}

1) B. Mori and H. Nakatsuji, Agric. Biol. Chem., 41, 345 (1977).

2) B. Mori, S. Saito and K. Kojima, Eiyo To Shokuryo, 30, 297 (1977).

3) E. W. Yemm and E. C. Cocking, Analyst, 80, 209 (1955).

4) K. Narita and T. Murakami, "Kuromatografii no Jittsusai," Vol. 1, Hirokawa Pub. Co., Tokyo, Japan, 1969, p. 212.

5) P. A. Finot and J. Mauron, Helv. Chim. Acta, 52, 1488 (1969).

6) C. H. Lea and R. S. Hannan, Biochim. Biophys. Acta, 5, 433 (1950).

7) B. Mori, K. Kojima and S. Saito, Abstracts of Papers, Annual Meeting of the Agricultural Chemical Society of Japan, Kyoto, April 1976, p. 150.

8) H. Erbersdobler, in "Protein Crosslinking-B (1977)," ed. by M. Friedman, Plenum Publishing Corporation, N. Y., 1977, p. 372.

9) H. Erbersdobler, I. Gunsser und G. Weber, Zentralbl. Veterinaermed., Reihe A, 17, 753 (1970).

10) P. A. Finot, in "Protein in Human Nutrition," ed. by J.W.G. Porter and B.A. Rols, Academic Press, N. Y., 1973, p. 507. 